\title{
Orthogonal cutting of cortical bone: Temperature elevation and fracture toughness
}

\begin{abstract}
During surgical procedures, the heat development of bone cutting can lead to thermal cell necrosis and secondary implant instability. Therefore, fundamental knowledge on heat development and temperature control is crucial. This paper investigates the basic principles of the machining of cortical bone in an orthogonal cutting process. Cutting forces, temperature elevation and chip formation were measured in real time for two different rake angles and six different cutting depths. A non-linear relationship between cutting depth and cutting forces as well as temperature elevation was found. A linear correlation between cutting forces and temperature elevation of both bone chip and workpiece was determined $\left(R^{2}=0.8697\right)$ An increasing rake angle lowered cutting forces and temperature elevations significantly and was explained using a fracture mechanics approach. Additionally, a new method to calculate the fracture toughness of (quasi-)brittle materials from orthogonal cutting tests was introduced.

Keywords: Orthogonal cutting, cortical bone, temperature elevation, heat of cutting, fracture toughness
\end{abstract}

Preprint submitted to Elsevier

March 26, 2017

(C) 2017. This manuscript version is made available under the Elsevier user license http://www.elsevier.com/open-access/userlicense/1.0/ 


\section{Introduction}

Surgical tools like drill bits, burs and bone saws are frequently used in orthopaedic interventions. Those tools are often not optimized to reduce the heat of the surgical procedures or even not adapted for cutting bone. However, the heat development of bone cutting can induce thermal cell necrosis (death of cells) which might lead to secondary implant instability [1] or to the damage of surrounding structures (e.g. nerves [2]). To reduce this risk and improve the tool design of future surgical tools, it is necessary to achieve a deeper understanding of how cutting tool design and process parameters relate to cutting forces and temperature elevation.

In order to investigate the basic principles for the machining of bone material, the cutting process has been reduced to an orthogonal cutting operation. The orthogonal cut enables a detailed experimental investigation of the cutting zone where the edge of a cutting tool is used to cut through a material in a linear movement. Measurement of the cutting forces and temperature elevations, as well as observation of the chip formation provide a knowledge base for the optimization of tool design and process parameters. Overall process optimization results in decreased cutting forces and temperatures, improved surface qualities and tool life.

In order to analyze cutting forces and temperature elevations, high speed optical and infrared cameras are needed. Thermal camera measurements have been previously used to investigate and reduce the heat development of a drilling process in bone. 3] Additionally, the necessary emissivity constant of cortical bone has been formerly determined. [4]

Wiggins and Malkin [5] as well as Jacobs [6] were the first to investigate the cutting forces of orthogonal cutting of cortical bone with respect to the osteonal cutting direction, tool rake angle and cutting depth. They found a non-linear relationship between cutting depth and cutting forces as well as a strong dependency of cutting direction on cutting forces, in relation to the bone micro-structure. Regardless of cutting depth or direction, it was found that 
an increase in tool rake angle decreases the cutting forces. This information has already been used to improve the geometry of surgical drill bits [3. More recent investigations by Yeager [7, Plaskos [8] and especially by Sugita et al. [9] [10] found that the cutting mode changes from continuous to fracture chip type at a certain cutting depth. There is no known study on the temperature measurement of the orthogonal cutting of bone.

In the latest research on orthogonal cutting of cortical bone, Liao and Axinte [11 made a great contribution to classify and explain the different cutting modes which depend on the cutting depth. They distinguish three cutting modes: The shear cutting mode (SC) which is only found at small cutting depths $(\leq 20 \mu m)$ without fracture due to the small cutting forces. In the shear-crack cutting mode (SCC) $(20 \sim 80 \mu \mathrm{m})$, the shear stress concentration along the presumed shear plane initiates a Mode II crack that runs from the top surface of the chip to the tip of the cutting tool along the shear plane. At an even greater cutting depth, the cutting mode changes to a so called fracture cutting mode (FC) in which the chip is fractured into larger pieces which appears to be close to a Mode I fracturing process.

The analytical modeling of the orthogonal cutting process of metals was first established by Ernst and Merchant in 1941 [12. They developed the shear plane model to describe and predict cutting forces for orthogonal metal cutting. Even though there has been numerous modifications (shear zone, slip-line, etc.) and it was found to be inadequate for many applications as described by Astakhov [13, it remains a widely used model for orthogonal cutting of ductile materials. The latest advancements in modeling orthogonal cutting have been summarized by Arrazola et al. 14] which include analytical, mechanistic, empirical or hybrid methods often calculated with FEM but limited to certain applications. Recent advances in numerical modeling of bone cutting have been summarized by Marco et al. 15. but there are only limited models for the cutting of brittle or quasi-brittle materials [16].

However, compared to metals, cortical bone is a very different material with a quasi-brittle and not ductile behavior. It is anisotropic with osteons $(\varnothing 200 \mu \mathrm{m})$ 
acting as "fibers" in a rather brittle matrix. Cortical bone has different intrinsic mechanisms to prevent crack growth which leads to a high fracture toughness that depends on the direction of the cutting process with respect to the osteonal structure.

Overall, the aim of this research is, for the first time, the investigation of the thermal increase in the orthogonal cutting process of cortical bovine bone and its relationship to cutting forces depending on the tool design and cutting depth in order to improve future surgical tool development and to reduce thermal cell necrosis. Additionally, we introduce a new method to calculate the fracture toughness of brittle or quasi-brittle materials using orthogonal cutting tests.

\section{Materials and Methods}

\subsection{Experimental Setup}

A custom test bench was used to conduct the experiments (at Fraunhofer Institute for Production Technology). It consists of a hydraulic linear slide carrying a specifically prepared workpiece (specimen). The linear slide allows the acceleration of the workpiece up to a velocity of $v_{c}=140 \mathrm{~m} / \mathrm{min}$. The orthogonal cutting operation is then performed by moving the workpiece against a cutting insert. The cutting insert is mounted to the height adjustable platform, which allows precise adjustment of cutting depths, and the granite bed of the test bench via a force measuring platform (Kistler Type 9129AA; Sampling Rate $10 \mathrm{kHz}$ ). Stiffness and accuracy of the setup is achieved by granite foundation and hydraulic linear slide 


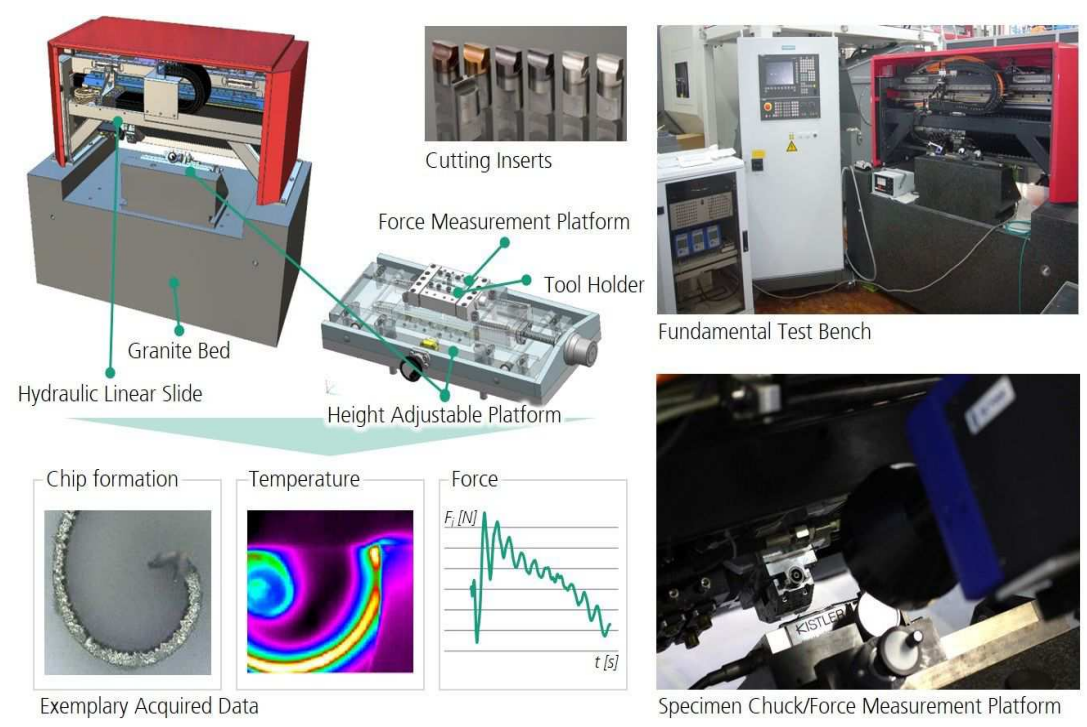

Figure 1: Experimental setup for orthogonal cutting experiments. Cutting forces, temperature elevation and high speed videos can be obtained.

The setup allows for the systematic investigation of different tool types (micro geometry, substrate, coating, etc.), different workpiece materials (metals, ceramics, fiber-reinforced materials, etc.) and process parameters (cutting velocity, cutting depths, etc.). Furthermore, the setup provides an excellent accessibility of the chipping zone, which allows for a detailed investigation of the chip forming behavior, the temperature elevations and the cutting forces. High-speed-imaging is performed by a high-speed-camera (DRS Lightning RDT PlusTM; Frame rate 6,000 fps; Frame size 512x424 pixel) while temperature measurements are done using thermal imaging (FLIR X6580sc; Frame rate 574 fps; Frame size 640x300 pixel; Integration time 0.506 ms; Emissivity 0.96 [4]).

\subsection{Sample Preparation}

Bovine cortical bone samples were extracted from fresh femur and tibia of dairy cows older than four years acquired from a local slaughterhouse. They were pre-cut using a hand saw and further prepared to their final dimension of $3 \mathrm{~mm}$ x $20 \mathrm{~mm}$ x $25 \mathrm{~mm}$ using a diamond band saw (Exakt, Germany). The long edge of the sample $(25 \mathrm{~mm})$ is thereby orientated parallel to the 
osteonal axis of the bones as shown in Figure 2. Additionally, a thin slice of bone was cut from each sample perpendicular to the the axial orientation (30 $\mathrm{mm} \times 20 \mathrm{~mm} \times 0.5 \mathrm{~mm}$ ). These slices were than checked for primary osteonal structures and sorted out if lamellar bone was found. In most cases, the osteonal structure was only found in the middle part of the diaphysis because of the highest stresses [17]. In fast growing bovine bones, the lamellar structure is only slowly remodeled within the life span of around 20 years, however, dairy cow bones are the oldest commercially available bones. In total, 25 osteonal bone samples were produced. It will be discussed that there is a clear difference between primary and secondary osteons which has been so far neglected in the literature. The bone samples were kept at $-20^{\circ} \mathrm{C}$ and thawed in saline solution prior to experiments.

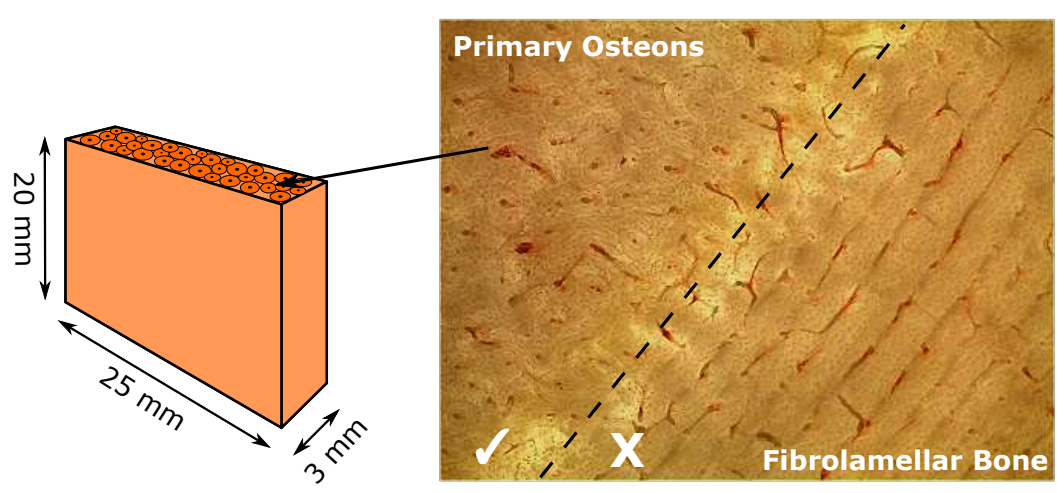

Figure 2: Dimension of cortical bovine bone sample in the transverse orientation. Only primary osteonal bone was used.

\subsection{Experimental procedure}

The experimental procedure was designed to investigate how tool rake angle and cutting depths affect the cutting force and temperature during bone cutting. Therefore, tungsten carbide tools (WC-10\%Co; uncoated) with two different rake angles $\left(\gamma=10^{\circ}\right.$ and $\left.40^{\circ}\right)$ but the same clearance angle $\left(\alpha=15^{\circ}\right)$ and a small cutting edge radius $\left(r_{\text {beta }}=5 \mu \mathrm{m}\right.$ ) were selected (see Figure 3 for nomenclature). The width of the tools $(5 \mathrm{~mm})$ transverse to the cutting direction was larger 
than the sample's width. Five tools were used for each rake angle and changed within the experiments to avoid excessive tool wear.

The average cutting depths of surgical tools like drill bits is up to ca. $50 \mu \mathrm{m}$ while a non-linear cutting behavior has been reported for larger cutting depths reported in the literature. Therefore, six cutting depths were chosen $(\mathrm{h}=12.5$, $25,50,75,100$ and $150 \mu \mathrm{m})$ to investigate the changes in cutting mode. The cutting velocity was set to $v_{c}=8 \mathrm{~m} / \mathrm{min}$ to represent the average speed of surgical cutting tools (e.g. standard surgical drill bit, $\varnothing 3 \mathrm{~mm}, 1000 \mathrm{RPM}$ ).

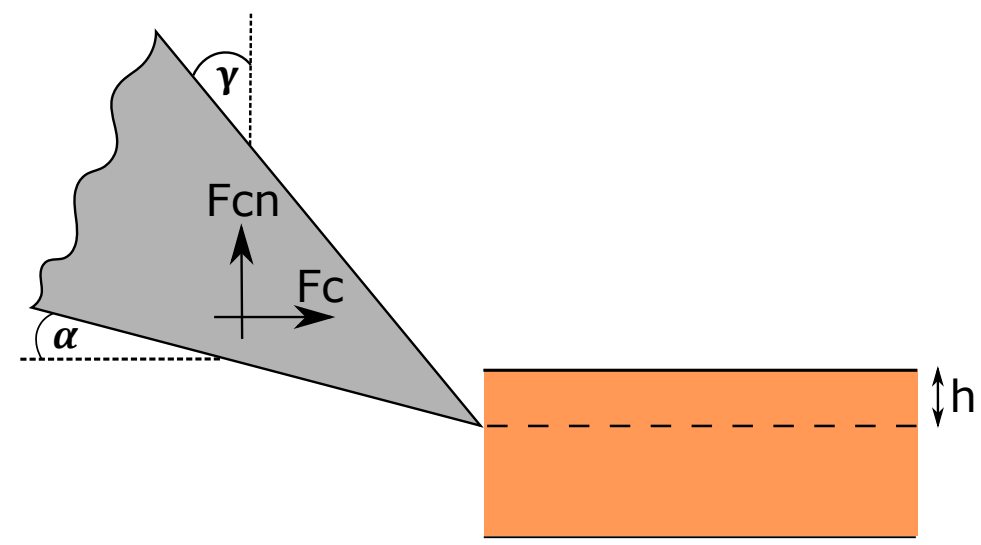

Figure 3: Definition of tool rake angle $(\gamma)$, clearance angle $(\alpha)$ and unreformed cutting depth (h).

Experiments were conducted for both cutting tools at all cutting depths and repeated three times for temperature measurements and one time for high speed camera videos. To ensure a smooth and planar surface, the bone samples were pre-cut and the surface topography was measured with a built-in measuring device. Afterwards, the sample was cut and the surface height was measured again so that set and actual cutting depths were available. Multiple operations have been performed using one sample, but the samples were randomized so that repetitions were not done on the same sample.

The cutting force was measured parallel $\left(F_{c}\right)$ and normal to the workpiece surface $\left(F_{c n}\right)$ as shown in Figure 3 . The sample width $(\mathrm{w})$ is measured for each sample (circa $3 \mathrm{~mm}$ ). Mean, minimal and maximal average cutting forces were 
extracted from the time interval of the cut which showed a continuous cutting behavior. Additionally, the averaged maximal temperature elevations of the bone chip and work piece were extracted from the same time interval as used for the cutting forces using the Flir ResearchIR software (Flir, USA). The region of temperature extraction was defined close to the rake face of the cutting tool and on the work piece surface directly behind the tip of the cutting tool.

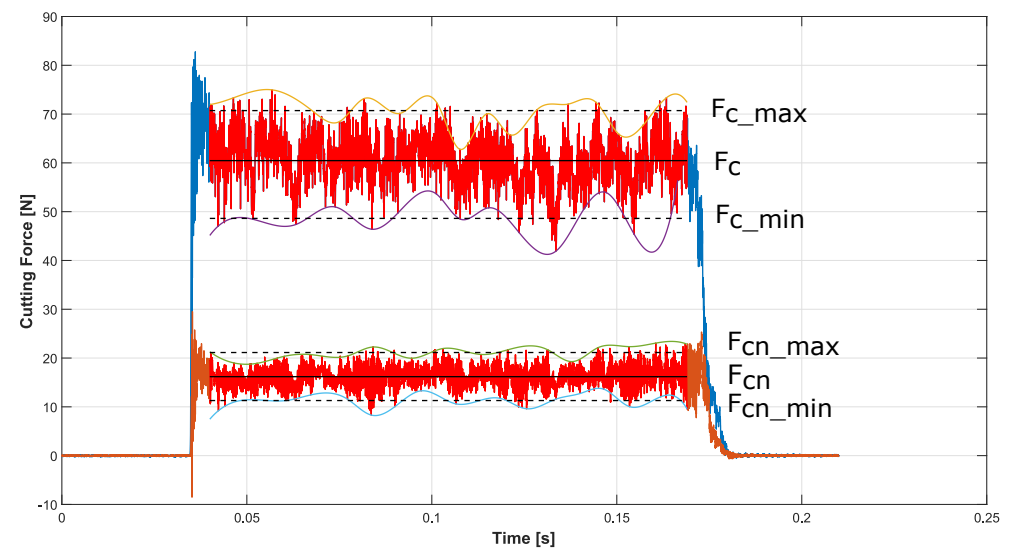

Figure 4: The force (and temperature; not shown here) data was extracted from the regions of continuous cutting behavior. The mean, maximal an minimal cutting forces were averaged for both measurement directions.

\section{Results}

An overview of the high speed optical and thermal camera images for different cutting depths and the two different rake angles is given in Figure 5 . The three cutting modes (as described in [11]) can be clearly distinguished and are visible for both cameras. However, obvious differences are also visible for the two different rake angles which will be discussed in great detail. Only four of the six cutting depths are shown because the cutting behavior at $75 \mu \mathrm{m}$ is similar to $50 \mu m$ and $100 \mu m$ to $150 \mu m$ cutting depth, respectively. 


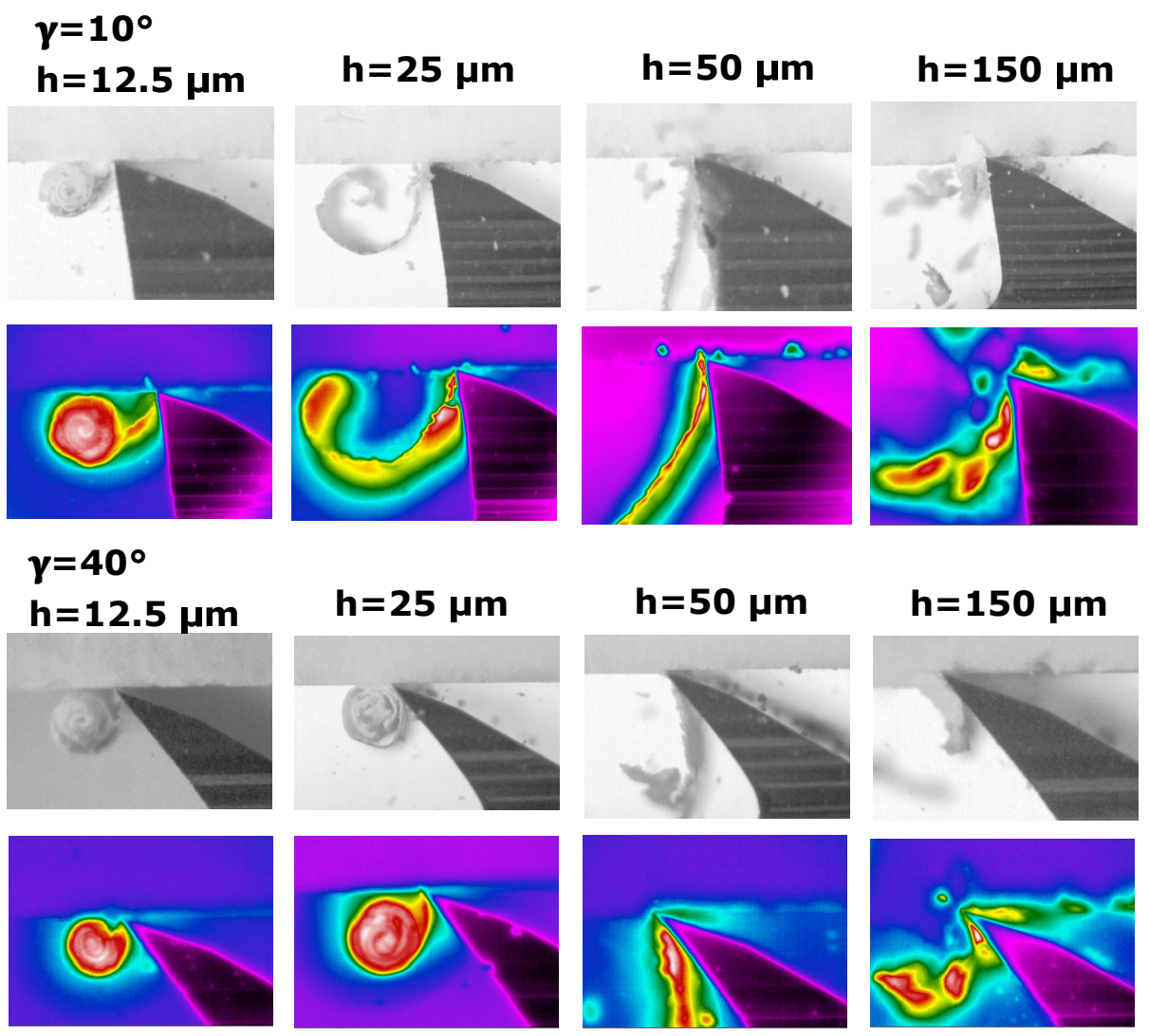

Figure 5: Influence of cutting depth $(\mathrm{h}=12.5-150 \mu \mathrm{m})$ and tool rake angle $\left(\gamma=10^{\circ}-40^{\circ}\right)$ on the cutting behavior and the temperature elevation in bone cutting. The color-scale of the thermal camera images is approximately between $20^{\circ} \mathrm{C}$ and $50^{\circ} \mathrm{C}$

\subsection{Cutting forces}

Figure 6 shows the measured cutting forces divided by the samples width $(\mathrm{w})$ in cutting direction $\left(F_{c}\right)$ and normal to the cutting direction $\left(F_{c n}\right)$ for all samples over the measured cutting depths. The graphs for $F_{c}$ over h are not linear as it would be expected for metals but have a more square root function shape as reported in the literature $[5$. The cutting forces are higher for the tool with the lower rake angle $\left(\gamma=10^{\circ}\right)$. The cutting forces in the normal direction are lower and even negative for $\gamma=40^{\circ}$. Two "outliers" were found $\left(\gamma=10^{\circ}, \mathrm{h}\right.$ $=75 \mu \mathrm{m}$, red circles in Figures) with both values coming from the same sample. 


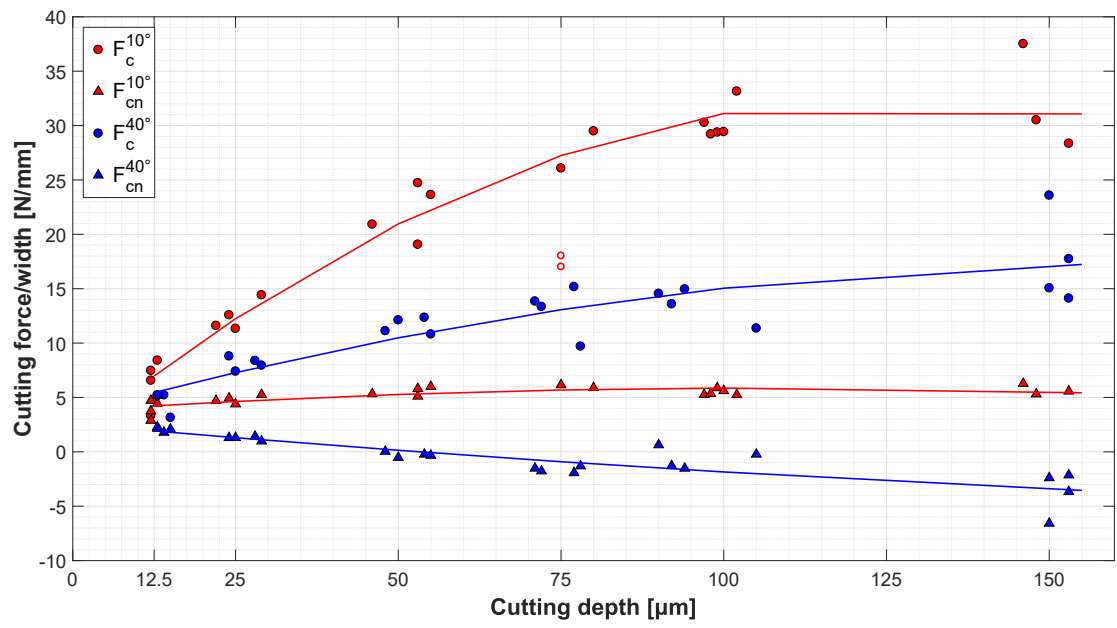

Figure 6: Cutting forces $\left(F_{c}\right.$ and $\left.F_{c n}\right)$ divided by the sample width (w) over cutting depths (h). A non-linear increase of cutting forces with cutting depth was found for both rake angles. The tool with the lower rake angle $\left(\gamma=10^{\circ}\right)$ created significantly higher cutting forces.

If the values in Figure 6 are divided by the cutting depth, the so called specific cutting force $\left(F_{c s p}\right)$ can be calculated as shown in Figure 7 . It represents the amount of cutting force per unit volume needed to remove the material. Analog to Figure 6, this graph shows a non-linear dependency with increasing cutting depth which results in decreasing cutting forces. It can also be seen that a larger rake angle significantly lowers the specific cutting forces needed to remove the material $(\sim 30 \%$ less force needed $)$. 


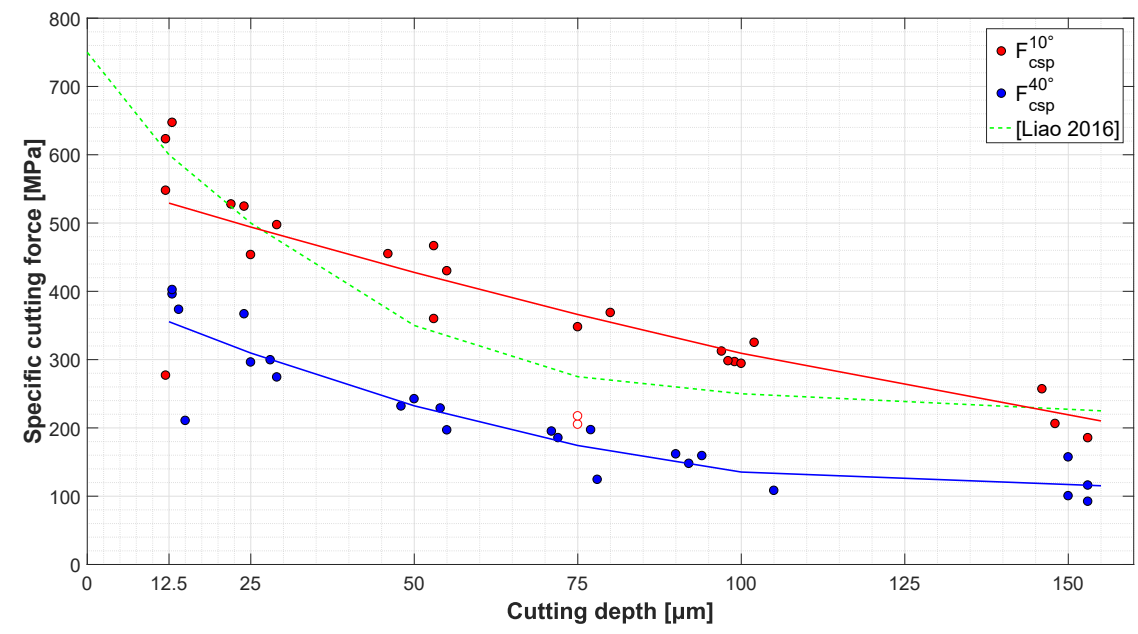

Figure 7: The specific cutting force is the amount of cutting force per unit volume needed to remove the material. A larger rake angle and higher cutting depth lowers the required specific cutting force. The green dotted line is a comparison with values obtained by Liao et al.

\subsection{Temperature elevation}

Figure 8 summarizes the results of the temperature measurements with respect to cutting tools and cutting depths. Both temperature elevation in the bone chip and the work piece were measured. The temperature is higher in the bone chips than the work piece and lower for the tool with the higher rake angle. In general, the curve looks similar to the cutting force $\left(F_{c}\right)$. 


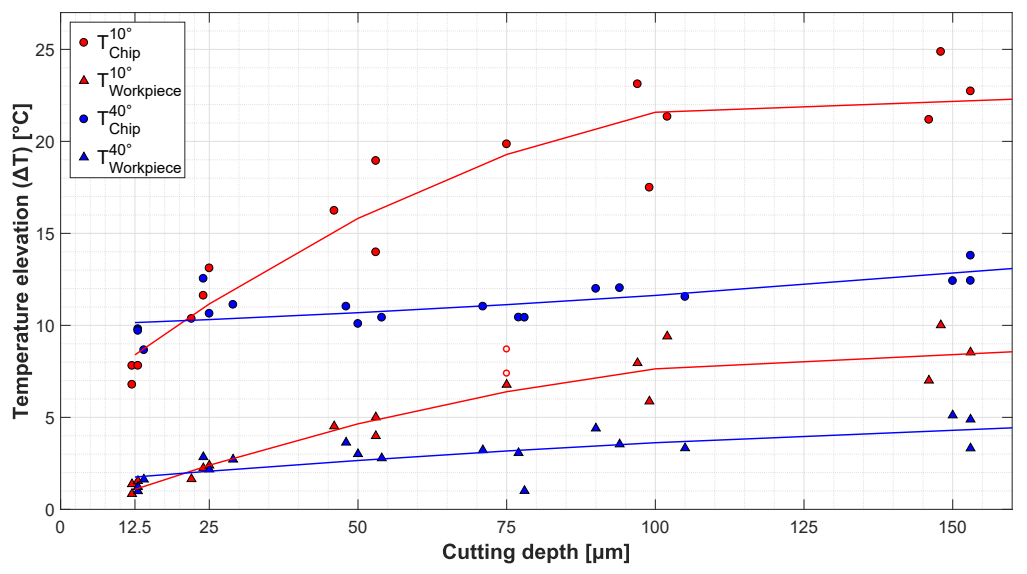

Figure 8: The temperature elevation of both bone chip after removal and work piece temperature are shown for both tools and all cuttings depths. The temperature elevation in the chip is significant higher and over all are the temperatures lower for the higher rake angle $\left(\gamma=40^{\circ}\right)$

To address the important issue how cutting forces relate to temperature elevations, in Figure 9 cutting force $\left(F_{c}\right)$ are plotted against the temperature of the bone chips. The linear regression shows a strong dependency with $R^{2}=$ 0.8512 and $S E E=1.958^{\circ} \mathrm{C}$ (standard error of the estimates) for the bone chip and $R^{2}=0.8556 S E E=0.9394^{\circ} \mathrm{C}$ for the work piece.

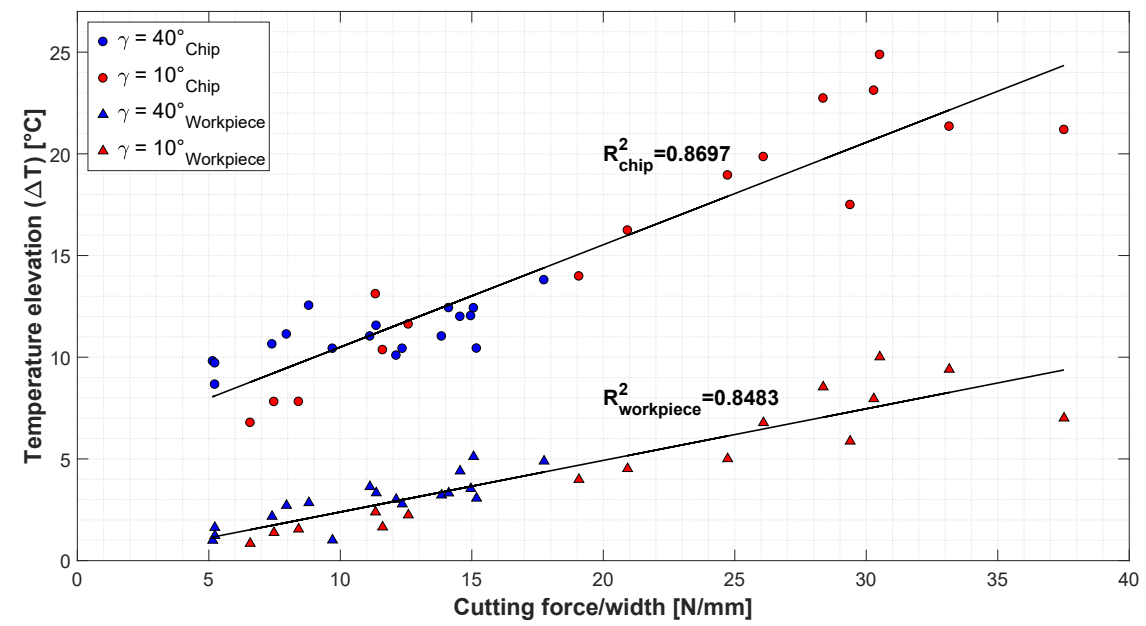

Figure 9: The linear regression of temperature rise with cutting force/width $\left(F_{c} / w\right)$ shows a high correlation for both bone chips and work piece temperature with cutting forces. 


\subsection{Chip formation}

As mentioned in the introduction, Liao et al. 11] summarized the different mechanisms of chip formation in cortical bone. They found that the differences of maximum and minimum cutting forces $F_{c}\left(\Delta F_{c}\right.$; see Figure 5$)$ are indicators for the different cutting modes (shear cutting, shear crack cutting and fracture cutting). A similar pattern was found for this experiment regardless of the rake angle as seen in Figure 10.

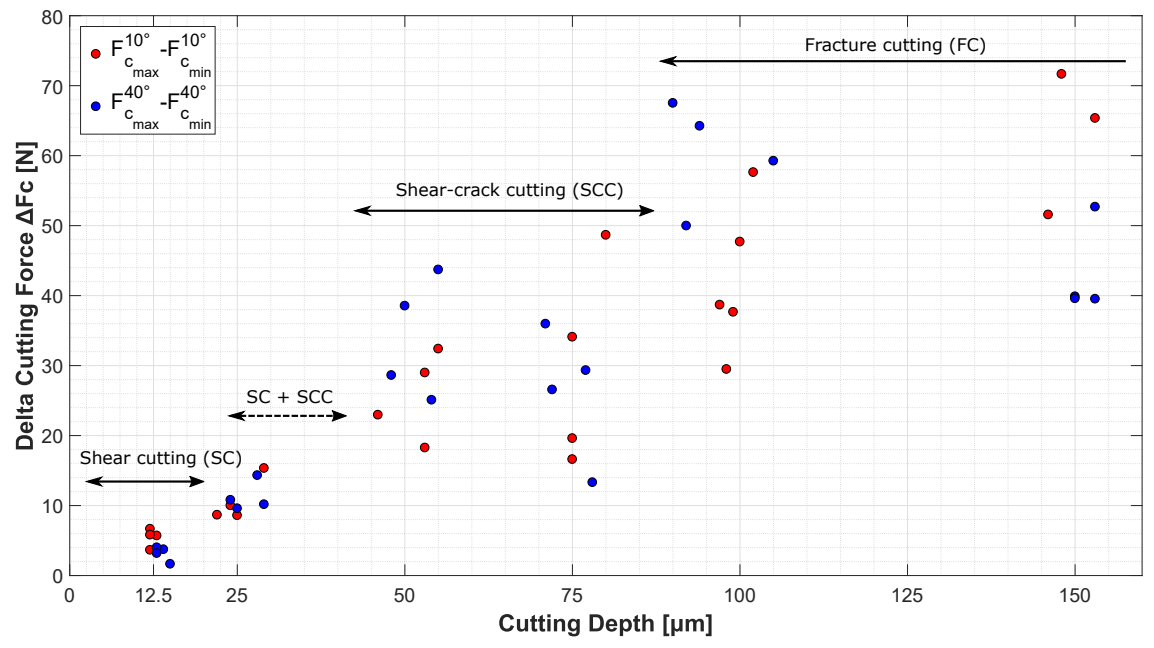

Figure 10: The difference of maximal and minimal cutting forces $\left(\Delta F_{c}=F_{c \max }-F_{c m i n}\right)$ can be used to distinguish the three cutting modes as suggest by Liao et al. [11.

Those findings were confirmed with scanning electron microscope images (Zeiss DSM 982, Germany) of the bone chips. Bone chips from each cutting mode $\left(\gamma=40^{\circ}\right)$ were acquired as shown in Figure 11. At the smallest cutting depth $(12.5 \mu \mathrm{m})$ the bone chips appear plastically deformed and rolled into a spiral. The outer surface is very smooth with no signs of cracks while the inner side of this chip type shows signs of compression and bending.

In the shear-cracking mode, the bone chips are fractured in constant repetitive intervals along a shear plane. At a deeper cutting depth, they are fractured into larger parts of rather irregular shape and cracks running in different directions. 


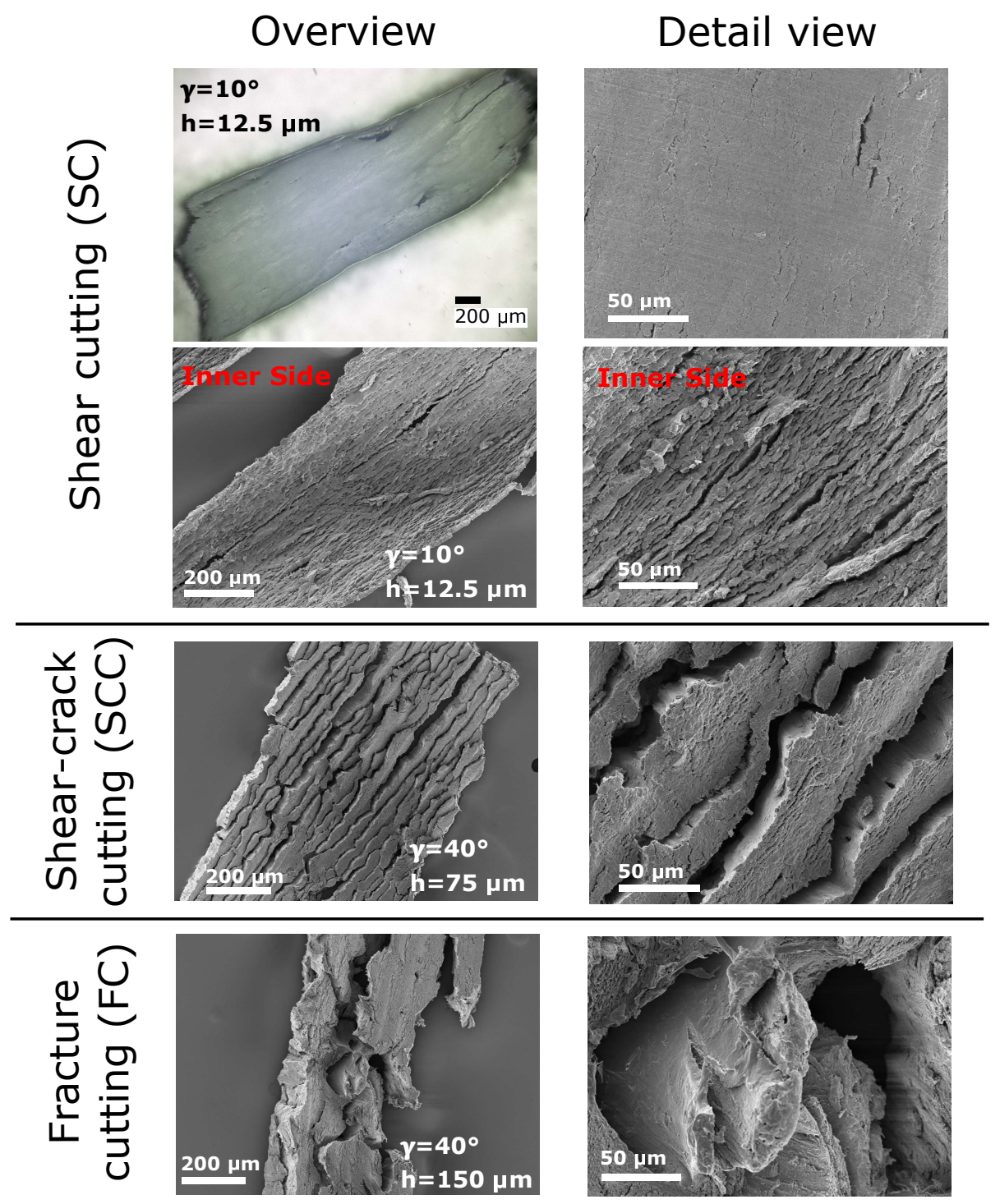

Figure 11: The SEM analysis of the bone chips for the three cutting modes highlights the differences of no fracture propagation (shear cutting), fracturing along the shear plane (shearcrack cutting) or fracturing of larger pieces ahead of the cutting tool (fracture cutting). 


\section{Discussion}

For the first time, both cutting forces and temperature elevation of orthogonal cutting experiments of cortical bone were measured. The cutting speed, cutting depth, cutting width and tool geometry were chosen to match common surgical tools (e.g. drill bits) and process parameters. Bovine cortical bone samples with primary osteons were selected and cut perpendicular to the main axis (transverse direction). This direction was chosen because of the expected highest forces of all three cutting directions.

The measured values were compared (see Figure 7) with the most recent work on orthogonal cutting of bone from Liao et al. [11. Their process parameters are different $\left(v_{c} \approx 2 \mathrm{~m} / \mathrm{min}\right.$; sample width $\left.1 \mathrm{~mm} ; \gamma=8^{\circ}\right)$ but the values of the tool with $\gamma=10^{\circ}$ shows fair agreement. The non-linear relationship between cutting forces and cutting depth of cortical bone is known for a long time but was never sufficiently explained. Our results suggest that this is due to a change of the failure mode as it was previously mentioned for PMMA and Nylon-66 by Wyeth and Atkins [18. At small cutting depths, bone is cut in a ductile fashion with a fracture process only at the tip of the tool in the direction of the cut surface which is needed for material separation [16]. Therefore, no cracks can be seen in the microscope images of the continuous curly chips (Figure 11 Shear cutting mode). However, most of the energy is used for plastic deformation of the bone chips so that this cutting behavior can be compared to metal cutting. At larger cutting depths, the material separation is a pure cracking process. The reason for the non-linear force-depth correlation is therefore that a plastic deformation is dependent on the volume deformation, but a cracking process only on the surface area of the crack (as seen in Figure 6 cube/square scaling law). In other words, the transition from continuous chip-type to segmented chip-type depends on the stress-strain behavior of the material in shear which makes the cutting process more efficient. In the shear cutting mode (low cutting depth), the differences in cutting forces are rather small which could be due to the similarities in the ductile cutting mode or due to the larger influence of the 
cutting edge radius on the cutting process.

As investigated by Liao et al. [11, the three cutting modes (shear cutting, shear-crack cutting and fracture cutting) can be distinguished by analyzing the differences of maximum and minimum cutting forces $\left(\Delta F_{c}\right)$ as seen in Figure 10. This method works well regardless of the rake angle. The three cutting modes were confirmed by the SEM image analysis.

a)
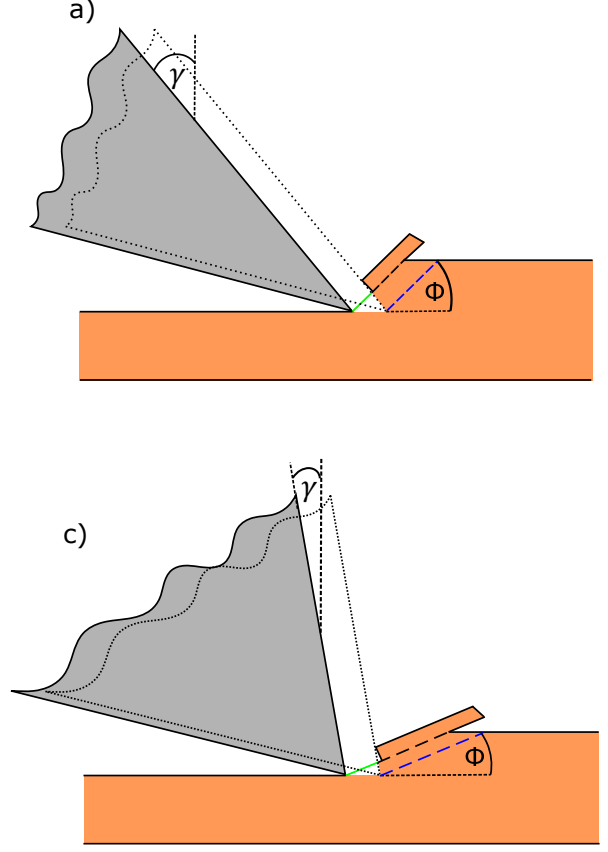

b)
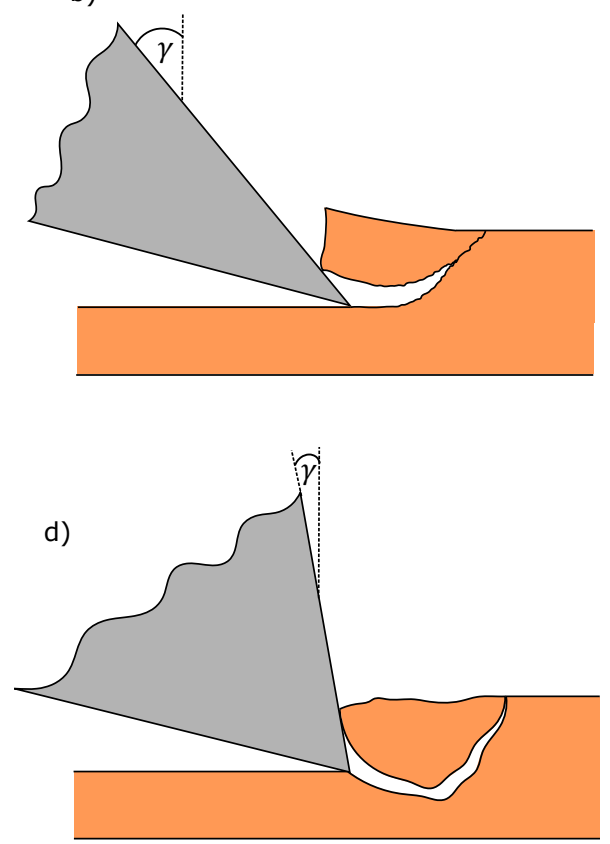

Figure 12: The shear-crack cutting mode a) and c) and fracture cutting mode b) and d) are schematically drawn for both cutting tools. A higher rake angle $(\gamma)$ leads to a higher shear angle $(\phi)$ according to Ernst and Merchant [12. Therefore the tool with the higher rake angle has a smaller shear plane and needs lower shear forces to cut the material in the shear-crack cutting mode. The difference in the fracture cutting mode are more complex, but the higher rake angle tool might function as a wedge for a Mode I cracking mode while a lower rake angle initiates a shear and might therefore drive the crack into the work material before it turns upwards.

In the shear-cutting mode, once the shearing forces overcome the fracture toughness of the material (Mode II), the bone fractures along the presumed shear plane. Liao et al. [11] suggest that this crack initiation starts at the free surface and runs towards the tip of the tool. Due to the higher cutting speeds, this could not be evaluated in this experiment. Figure 12 shows that after crack 
initiation, the further tool movement pushes the broken chip upwards until another shear crack is initiated in a parallel shear plane.

The schematic representation shows however, that there are differences depending on the rake angle. In the shear-crack cutting mode, the shear plane angle $(\phi)$ is according to Merchants [12] or other metal cutting theories dependent on the rake angle which leads in general to a larger shear angle with larger rake angle [12. The cutting tool with the rake angle of $10^{\circ}$ has therefore also a larger shear plane which could be the reason for the higher encountered cutting forces. At larger cutting depths starting around $100 \mu \mathrm{m}$, the cutting mode changes to fracture cutting along a putative fracture direction parallel to the cutting direction. It is assumed that either the remaining cutting forces after the shearcracking are still high enough to initiate further cracks or that even without the shear plane fracture these cracks find weaker planes at which they travel. Additionally, it is more likely that weaker interfaces are found with increasing cutting depth. The difference to the shear-crack cutting is however that the initiation of the crack is not occurring on the free surface but starts at the tip of the cutting tool and runs ahead of the tool. From the high speed videos (Figure 5), it can be concluded that there are however major differences depending on the rake angle (Figure 12).

The higher rake angled tool is able to drive the initiated crack in a Mode I cracking process. It should be noted that the fracture toughness of Mode I was found to be smaller than Mode II [19]. The pointed tool acts like a wedge in wood wood cutting and the crack is therefore not driven deeper into the material but is able to turn upwards to the free surface. The tool with the lower rake angle $\left(10^{\circ}\right)$ rather shears the bone which could lead to a crack deviation into the work material before it turns upwards again. This is also supported by the fact that the surface roughness of the workpiece is higher at a large cutting depth with a lower rake angled tool [7]. It is most likely that both cutting modes are a mix between Mode I and Mode II. The ratio of this mix could be dependent on the shear angle (and therefore rake angle) as suggested by Wyeth [18, with a larger rake angle corresponding to a larger part of Mode I fracturing. 
A further indication of the fracture mode has been found by analyzing the force signal in greater detail. Figure 13 shows the comparison between the different cutting modes depending on cutting depth or rake angle. Due to the high sampling rate of the load cell and the very stiff hydraulic orthogonal cutting setup, it is possible to analyze the cutting force signals in greater detail. As discussed above, the differences of maximal and minimal cutting forces correspond to the cutting modes. As seen in Figure 13 a) and b), not only the amount of cutting forces but also the time intervals $\left(\Delta t_{1}, \Delta t_{2}\right)$ which are needed for fracturing one chip are very different. In the shear-cracking mode (blue line), the cracking process is very repetitive in the shear plane while in the fracture mode, the crack travels ahead of the tool. Comparing the force signals for the two different rake angles at the largest cutting depth (c), the force signal is a further indicator for the differences in the fracture process. The larger amplitude and time interval length to fracture was found for the $\gamma=10^{\circ}$ tool. A reason for this could be that the crack is driven into the work material for $\gamma=10^{\circ}$ while the crack travels rather horizontally before it turns upwards for $\gamma=40^{\circ}$. 


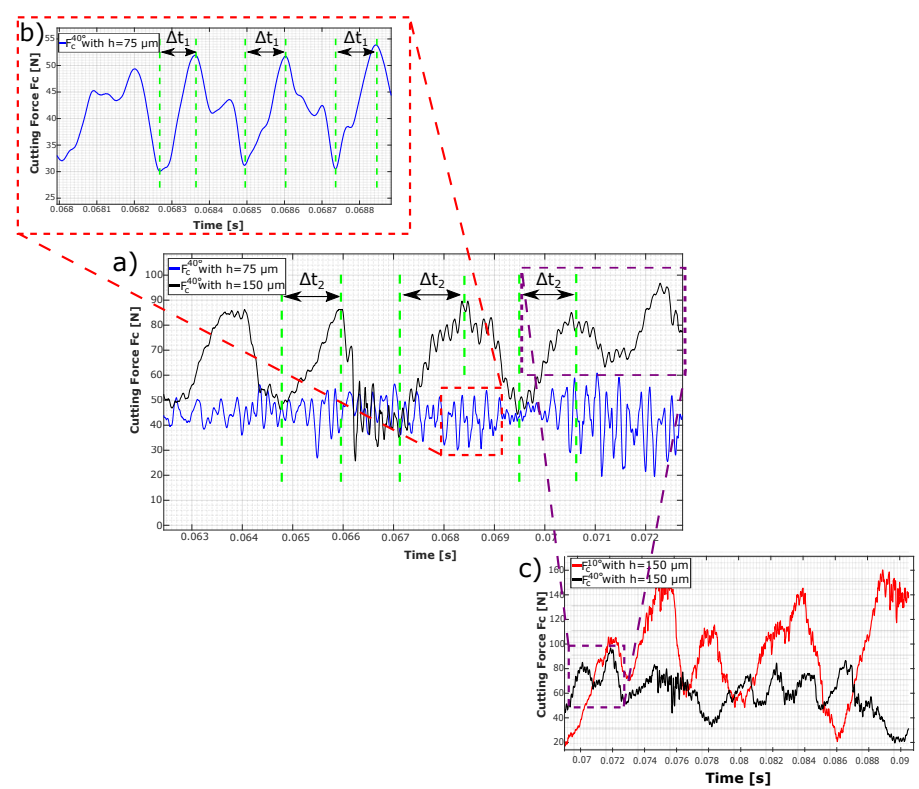

Figure 13: A detailed analysis of the force signal $\left(F_{c}\right)$ can help to understand the different fracture patterns. a) Comparison of force signals for a cutting depth of $75 \mu \mathrm{m}$ and $150 \mu \mathrm{m}$ for $\gamma=40^{\circ}$. b) Detailed view of force pattern at $75 \mu \mathrm{m}$ shows a periodical rise and fall of the signal which corresponds to a repetitive fracturing along the shear plane. c) Highlights the differences of the force signal for tools with the two different rake angles at the highest cutting depth $(150 \mu \mathrm{m})$ : The lower rake angle tool needs apparently more time to cut out one bone chip from the material (=longer crack travel time).

The fact that cortical bone is an anisotropic material amplifies the described effects and leads to crack deviation along cement lines as described by Zimmermann et al. [20]. In the transverse direction, which leads to the highest cutting forces, the cracks are more prone to deviate up and downwards, while in the other two cutting directions the crack can more easily travel in the cutting direction in-between osteons. It should however be noted that the investigated bovine cortical bone has a primary osteonal structure which clearly needs to be distinguished from secondary osteons (Haversian system) [17. Primary osteons do not have a cement layer and have therefore a less developed anisotropic effect on fracture travel. This has not been discussed in previous literature on orthogonal cutting of cortical bone but it is assumed that no bovine bone samples have secondary osteons due to the difficulty in acquiring aged bovine bones 
(>15 years). Wiggins et al. [5] however found similar cutting forces in human and bovine bone.

The temperature elevation of both bone chips and workpiece correlate well with cutting forces. The slightly higher temperature found for a rake angle of $40^{\circ}$ at the smallest cutting depth is most likely due to difficulties in temperature readout of the very curled chip and accumulating temperature effect. In general, the temperature rise of the workpiece is however much lower than of the chip which suggests that most of the heat is transferred to the chip similar to metal cutting [21].

These findings can be important for future surgical tool development. Even though the drilling process is much more complex than orthogonal cutting, a few basic relationships were found. On the one hand it was confirmed that a higher rake angle leads to lower cutting forces and therefore lower temperature elevation. The lower temperature could preserve osteblasts or nerve cells which leads to a safer surgical procedure. Additionally, cutting at larger cutting depths are more efficient but might lead to a higher surface roughness. It has however not been investigated yet if a higher surface roughness might actually be beneficial for, for example, bone implant in-growth. 


\subsection{Fracture toughness}

The authors would also like to discuss the topic of fracture toughness determination from orthogonal cutting tests which has recently gained a lot of attention. The advantage of such test are that they are potentially fast and easier and have less restrictions on sample dimensions. Atkins [22] was the first to acknowledge that traditional models of metal cutting which mainly base on Merchants shear plane model neglect the work needed for the formation of new surfaces. Even though this idea has been challenged [23, 24] many studies used orthogonal cutting test to find the fracture toughness of ductile materials [25, 26, 27, 28, 29, 30] and it was suggested that this might also be possible for cortical bone [15].

Due to the non-linearity of the cutting depth vs. force diagram and the unknown exact transition to fracture cutting it is not possible to use Atkins method. A second method should be mentioned which has been suggested by Akono and Ulm [31] to determine fracture toughness values of stones with scratch tests which are like orthogonal cutting tests with negative rake angle tool. In contrast to Atkins, they argue that the change of potential energy with fracture surface creation, as evaluated by the J-integral, leads to a quadratic relationship between fracture toughness and cutting forces. Therefore, even though also their method has been criticized [32, they state that the slope of the linear relationship between applied forces divided by the width of cut and the square root of cutting depth is actually the critical stress intensity factor (witch fits with the units). The found values for our measurements are indeed straight lines and found values $\left(K_{c}=2-4 M N m^{-\frac{3}{2}}\right)$ are close to reported literature values $\left(K_{c}=3-12 M N m^{-\frac{3}{2}}\right)$, but should not change with the rake angle. Wyeth et al. [18] and Atkins [16] wrote that those values are somehow connected to the critical stress intensity factor which is indeed controlling the cutting process, but that a not yet existing fracture mechanics solution for a beam of very short length is needed to predict the fracture toughness from cutting forces.

Wiggins [5] suggested a further method by correlating the surface area of the chips divided by the volume of the chip segments and correlated this ratio with 


\section{DISCUSSION}

the specific cutting force. He argues that if all the energy for chip formation is expended by primary fracture, the slope of the straight line of this relationship can be interpreted as the energy required to generate a unit surface area of chip segment by fracture. This however needs a very laborious analysis of the bone chips.

Liao et al. [11] used literature fracture toughness value to determine the cutting depth at which the transition of chip formation changes. If this method is reversed, the fracture toughness can be determined at the cutting depth but it has to be exactly known at which depth the bone chips fracture for the first time along the shear plane. Therefore the authors would like to suggest a new method which is independent of chip analysis and straight forward to calculate. The fracture toughness " $G_{c}$ " is the work needed for fracture divided by the fracture area as seen in equation 1. If its assumed that the fracture occurs along the shear plane, the work for fracturing is the shear force " $F_{s}$ " times the displacement " $\Delta s$ " along the shear plane. The crack area is equal to the shear plane " $A_{s}$ " because it travels all the way from the free surface towards the tip of the tool throughout the whole chip.

$$
G_{c}=\frac{d W}{d A}=\frac{F_{s} \cdot \Delta s}{A_{s}}
$$

" $F_{s} "$ (eq. 2 ) and " $A_{s} "$ (eq. 3) were calculated according to Ernst-Merchants shear plane model [12]. Though this is mainly a fracture and not a plastic deformation process, the model rather looks like Piispanens card model [33].

$$
\begin{gathered}
F_{s}=F_{c} \cos (\phi)-F_{c n} \sin (\phi) \\
A_{s}=\frac{w \cdot h}{\sin (\phi)} \\
\text { with } \phi=45^{\circ}+\frac{\gamma}{2}-\frac{\beta}{2} \text { and } \beta=\tan ^{-1}(\mu)=\tan ^{-1}\left(\frac{F_{c n}+F_{c} \tan (\gamma)}{F_{c}-F_{c n} \tan (\gamma)}\right)
\end{gathered}
$$


The remaining unknown displacement can be derived from the force signal as shown previously in Figure 13. It is assumed that the displacement needed for fracture " $\Delta s "$ is related to the time of the rising of the force signal. Figure 14 shows one cycle of an abstracted cutting force signal $\left(F_{c}\right)$ over time. Multiplying the time necessary to crack the bone " $\Delta t$ " with the speed of the cutting tool " $v_{c}$ " allows to calculate the displacement of the tool in cutting direction. The direction of the work is however along the shear plane which requires a projection onto onto the shear plane as used in equation 4.

$$
\Delta s=\Delta t \cdot v_{c} \cdot \frac{\sin (\delta)}{\sin (\varepsilon)}=\Delta t_{1} \cdot \frac{8}{60} \mathrm{~m} / \mathrm{s} \cdot \frac{\sin \left(90^{\circ}-\gamma\right)}{\sin \left(180^{\circ}-\left(90^{\circ}-\gamma\right)-\phi\right)}
$$

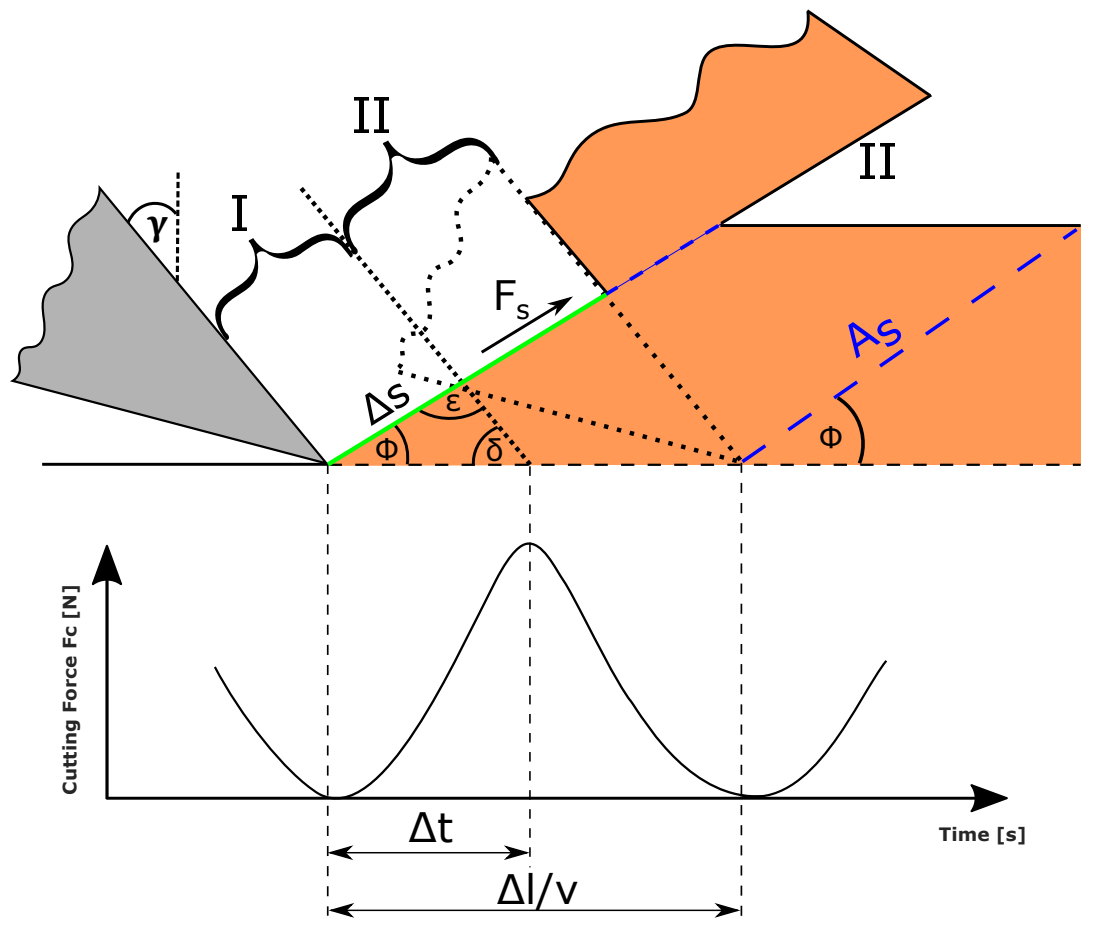

Figure 14: Detailed analysis of the shear-crack cutting mode. The rise of the force signal $\left(F_{c}\right)$ from Figure 13 corresponds to the displacement of the tool needed of the shear force $\left(F_{S}\right)$ to crack the material. The tool travel time is then projected onto the shear plane to calculate the fracture toughness. A further displacement of the tool pushes the bone chip upwards (falling force curve). This process repeats with a fracturing along the next parallel shear plane. 
The average values for the displacement time were extracted as $\Delta t_{1} \approx$ $1-2 * 10^{-} 4 \mathrm{~s}$ for $\gamma=40^{\circ}$ at a cutting depth of $\mathrm{h}=75 \mu \mathrm{m}$ as seen in Figure $13 \mathrm{~b}$ ). The shear force " $F_{s}$ " was calculated with $F_{c \max }$ and $F_{c n \max }$ as $F_{s} \approx$ $40 \mathrm{~N}$ and $\phi \approx 45^{\circ}$ when averaging all 4 repetitions for this tool and cutting depth. The displacement along shear plane was is therefore $\Delta s \approx 10-20 \mu \mathrm{m}$ with the cutting speed of $\mathrm{v}=8 \mathrm{~m} / \mathrm{min}$ and the shear plane size using the width of the sample as $\mathrm{w}=3 \mathrm{~mm}$ to be $A_{s} \approx 0.3 \mathrm{~mm}^{2}$. The calculated fracture toughness is therefore $G_{c} \approx 2000 \mathrm{~N} / \mathrm{m}$ which has the right dimension and is close to the reported values in literature for cortical bone for Mode II $\left(G_{c}=2000-5000\right.$ $\mathrm{N} / \mathrm{m}$ for longitudinal and transverse fracturing of bone [19]). Similar results were obtained for the tool with the smaller rake angle and for $\mathrm{h}=75 \mu \mathrm{m}$. Finally, the tool displacement after which the next shear fracture is occurring to be $\Delta l * v \approx 20-50 \mu m$ which corresponds well with the fracture pattern seen in the SEM images of the bone chips (Figure 11). This also allows to predict approximately the fracture pattern for the bone chips cut in the fracture mode (e.g. $\sim 2 * \Delta t_{2}$ in Figure 13 ).

This simplified testing method would allow to use orthogonal cutting test to determine the fracture toughness of brittle or quasi-brittle material. This requires however a stiff setup and a high sampling rate as well as known cutting depth region in which the materials fails along the shear plane. Furthermore the extraction of the correct and precise time interval is crucial for the calculation and should be facilitated in a constant and repetitive amplitude of the force signal. Additionally, it needs to be validated whether the fracture "shear" plane is actually correctly calculated using Ernst and Merchants formulas. The shear plane angle phi $(\phi)$ could also be calculated with Lee and Shafers 34 or Oxleys [35] slip-line theory or any other of the proposed analytical cutting models.

Finally, it should be noted that Zimmermann et al. [36] argues that only the rising R-curve is able to describe the fracture behavior of cortical bone because the majority of its toughness is derived during the crack growth and that Linear-Elastic-Fracture-Mechanics (LEFM) might only partly apply to bone. The calculated fracture toughness values could also be interpreted as initiation 
fracture toughness with the advantages of simplicity and more realistic (higher) strain rates. The results and methods could also be interesting for other materials like fiber-reinforced composites. Future studies on orthogonal bone cutting should facilitate a more complete analysis of the produced bone chips.

\section{Conclusion}

In this study, the cutting forces, temperature elevation and bone chip formation of orthogonal cutting tests of bovine cortical bone were investigated. Cortical bone was cut using two different tools (rake angle $=10^{\circ}$ and $40^{\circ}$ ) at six different cutting depths $(12.5-150 \mu \mathrm{m})$ in the transverse direction with respect to the micro-structure of the bone. The non-linear effects of cutting depth on cutting forces and temperature elevation were explained using a linear fracture mechanics approach. Three distinguishable cutting modes were found and the temperature elevation of chip $\left(R^{2}=0.8512, S E E=1.958^{\circ} \mathrm{C}\right)$ and work piece $\left(R^{2}=0.8483, S E E=0.9394^{\circ} C\right)$ were strongly correlated with the cutting forces. Overall, these findings suggest that high rake angle tools and large cutting depths significantly lower the cutting forces and temperature elevation. Those findings might help to improve the geometry and process parameters of surgical tools in order to reduce thermal necrosis of bone tissue. Additionally, a new approach to calculate the fracture toughness from orthogonal cutting tests was introduced which simplifies earlier attempts and allows to calculate the fracture toughness also for (quasi-)brittle materials.

\section{Acknowledgment}

The authors would like to thank Prof. Stoffel from the Veterinary Anatomy, Vetsuisse Faculty of the University of Bern for his help in acquiring the scanning microscope images. Additionally we would like to acknowledge support of the Swiss National Science Foundation for funding part of this research within the Nano Tera program [grant number 16-954]. 


\section{References}

[1] P. Trisi, G. Perfetti, Insufficient irrigation induces peri- implant bone resorption: An in vivo histologic analysis in sheep, Clinical oral implants research 25 (2014) 696-701. doi:10.1111/clr.12127.

[2] A. Feldmann, J. Anso, B. Bell, T. Williamson, K. Gavaghan, N. Gerber, H. Rohrbach, P. Zysset, Temperature Prediction Model for Bone Drilling Based on Density Distribution and In Vivo Experiments for Minimally Invasive Robotic Cochlear Implantation, Annals of Biomedical Engineering.

[3] A. Feldmann, J. Wandel, P. Zysset, Reducing temperature elevation of robotic bone drilling, Medical Engineering and Physics 0 (2016) 1-10. doi: 10.1016/j.medengphy.2016.10.001. URL http://dx.doi.org/10.1016/j.medengphy .2016.10.001

[4] A. Feldmann, P. Zysset, Experimental determination of the emissivity of bone, Medical Engineering and Physics (2016) 8-12.

[5] K. Wiggins, S. Malkin, Orthogonal machining of bone, Journal of Biomechanical Engineering 100 (1978) 122-130.

[6] C. H. Jacobs, M. H. Pope, J. T. Berry, A study of the bone machining process - Orthogonal cutting, Journal of Biomechanics 7 (1974) 131-136.

[7] C. Yeager, a. Nazari, D. Arola, Machining of Cortical Bone: Surface Texture, Surface Integrity and Cutting Forces, Machining Science and Technology 12 (1) (2008) 100-118. doi:10.1080/10910340801890961

URL http://www.tandfonline.com/doi/abs/10.1080/ 10910340801890961

[8] C. Plaskos, A. Hodgson, P. Cinquin, Modelling and optimization of bone-cutting forces in orthopaedic surgery, Medical Image Computing and and Computer Assisted Intervention (2003) 254-261.

URL

http://link.springer.com/chapter/10.1007/ 978-3-540-39899-8_32 
[9] N. Sugita, M. Mitsuishi, Specifications for machining the bovine cortical bone in relation to its microstructure, Journal of biomechanics 42 (16) (2009) 2826-9.

[10] J. Sui, N. Sugita, K. Ishii, K. Harada, M. Mitsuishi, Force Analysis of Orthogonal Cutting of Bovine Cortical Bone, Machining Science and Technology 17 (4) (2013) 637-649.

[11] Z. Liao, D. A. Axinte, On chip formation mechanism in orthogonal cutting of bone, International Journal of Machine Tools \& Manufacture 102 (2016) 41-55. doi:10.1016/j.ijmachtools.2015.12.004.

[12] H. Ernst, M. Merchant, Chip formation, friction and high quality machined surfaces. Surface treatment of metals, Am. Soc. Met., Surf. Treat. Met. 29 29 (1941) 299-378.

[13] V. P. Astakhov, On the inadequacy of the single-shear plane model of chip formation, International Journal of Mechanical Sciences 47 (11) (2005) 1649-1672. doi:10.1016/j.ijmecsci.2005.07.002.

URL http://linkinghub.elsevier.com/retrieve/pii/ S0020740305001864

[14] P. Arrazola, T. Özel, D. Umbrello, M. Davies, I. Jawahir, Recent advances in modelling of metal machining processes, CIRP Annals - Manufacturing Technology 62 (2) (2013) 695-718. doi:10.1016/j.cirp.2013.05.006. URL http://linkinghub.elsevier.com/retrieve/pii/ S0007850613001960

[15] M. Marco, M. Rodriguez-Millan, C. Santiuste, E. Giner, M. H. Miguelez, A review on recent advances in numerical modelling of bone cutting, Journal of the Mechanical Behavior of Biomedical Materials 44 (2015) 179-201.

[16] T. Atkins, The Science and Engineering of Cutting: The Mechanics and Processes of Separating, Scratching and Puncturing Biomaterials, Metals and Non-Metals by, Elsevier, 2009. 
[17] J. D. Currey, Bones: Structure and Mechanics, Princeton University Press, Princeton, 2006.

[18] D. Wyeth, a.G. Atkins, Mixed mode fracture toughness as a separation parameter when cutting polymers, Engineering Fracture Mechanics 76 (18) (2009) 2690-2697. doi:10.1016/j.engfracmech.2009.07.023

URL http://linkinghub.elsevier.com/retrieve/pii/ S0013794409002495

[19] Z. Feng, J. Rho, S. Han, I. Ziv, Orientation and loading condition dependence of fracture toughness in cortical bone, Materials Science \& Engineering C 11 (2000) 41-46.

[20] E. A. Zimmermann, M. E. Launey, H. D. Barth, R. O. Ritchie, Biomaterials Mixed-mode fracture of human cortical bone, Biomaterials 30 (29) (2009) 5877-5884. doi:10.1016/j.biomaterials.2009.06.017 URL http://dx.doi.org/10.1016/j.biomaterials.2009.06.017

[21] F. Klocke, W. König, Fertigungsverfahren 1 - Drehen, Fräsen, Bohren, 8th Edition, Springer, RWTH Aachen, 2008.

[22] A. Atkins, Modelling metal cutting using modern ductile fracture mechanics: quantitative explanations for some longstanding problems, International Journal of Mechanical Sciences 45 (2) (2003) 373-396. doi:10.1016/S0020-7403(03)00040-7.

URL http://linkinghub.elsevier.com/retrieve/pii/ S0020740303000407

[23] V. P. Astakhov, X. Xiao, A Methodology for Practical Cutting Force Evaluation Based on the Energy Spent in the Cutting System, Machining Science and Technology 12 (3) (2008) 325-347. doi:10.1080/10910340802306017 URL http://ww.tandfonline.com/doi/abs/10.1080/ 10910340802306017 
[24] T. Childs, Surface energy, cutting edge radius and material flow stress size effects in continuous chip formation of metals, CIRP Journal of Manufacturing Science and Technology 3 (1) (2010) 27-39. doi:10.1016/j.cirpj.2010.07.008

URL http://linkinghub.elsevier.com/retrieve/pii/ S1755581710000726

[25] D. Wyeth, An investigation into the mechanics of cutting using data from orthogonally cutting Nylon 66, International Journal of Machine Tools and Manufacture 48 (7-8) (2008) 896-904. doi:10.1016/j.ijmachtools.2007.11.001.

URL http://linkinghub.elsevier.com/retrieve/pii/ S0890695507002428

[26] Y. Patel, B. Blackman, J. Williams, Determining fracture toughness from cutting tests on polymers, Engineering Fracture Mechanics 76 (18) (2009) 2711-2730. doi:10.1016/j.engfracmech.2009.07.019.

URL http://linkinghub.elsevier.com/retrieve/pii/ S0013794409002446

[27] Y. Patel, B. Blackman, J. . Williams, Measuring fracture toughness from machining tests, Proceedings of the Institution of Mechanical Engineers, Part C: Journal of Mechanical Engineering Science 223 (12) (2009) 2861-2869. doi:10.1243/09544062JMES1497.

URL http://pic.sagepub.com/lookup/doi/10.1243/ 09544062JMES1497

[28] B. Wang, Z. Liu, Q. Yang, Investigations of yield stress, fracture toughness, and energy distribution in high speed orthogonal cutting, International Journal of Machine Tools and Manufacture 73 (2013) 1-8. doi:10.1016/j.ijmachtools.2013.05.007

URL http://linkinghub.elsevier.com/retrieve/pii/ S0890695513000874 
[29] B. Blackman, T. Hoult, Y. Patel, J. Williams, Tool sharpness as a factor in machining tests to determine toughness, Engineering Fracture Mechanics 101 (2013) 47-58. doi:10.1016/j.engfracmech.2012.09.020

URL http://linkinghub.elsevier.com/retrieve/pii/ S0013794412003827

[30] B. Blackman, J. Williams, T. Hoult, Y. Patel, Crack Tip Sharpness Effects in Fracture and Machining, ECF18, Dresden 2010 (2013) 2-7.

URL http://www.gruppofrattura.it/ocs/index.php/esis/ECF18/ paper/download/6074/1959

[31] A. Akono, Scratch Tests : A New Way of Evaluating the Fracture Toughness of Materials, Ph.D. thesis, Massachusetts Institute of Technology (2009).

[32] J. Lin, Y. Zhou, Can scratch tests give fracture toughness?, Engineering Fracture Mechanics 109 (2013) 161-168. doi:10.1016/j.engfracmech. 2013.06 .002

URL http://dx.doi.org/10.1016/j.engfracmech.2013.06.002

[33] V. Piispanen, Theory of formation of metal chips, Journal of applied physics (19) (1948) 876-881.

[34] E. Lee, B. Shaffer, The theory of plasticity applied to a problem of machining, Journal of appied Mechanics 18 (405-413).

[35] N. Fang, I. Jawahir, P. Oxley, A universal slip-line model with non-unique solutions for machining with curled chip formation and a restricted contact tool, International Journal of Mechanical Sciences 43 (2) (2001) 557-580. doi:10.1016/S0020-7403(99)00117-4.

URL http://linkinghub.elsevier.com/retrieve/pii/ S0020740399001174

[36] E. Zimmermann, M. E. Launey, R. O. Ritchie, The significance of crack-resistance curves to the mixed-mode fracture toughness 
of human cortical bone. Biomaterials 31 (20) (2010) 5297-305.

doi:10.1016/j.biomaterials.2010.03.056

URL http://www.pubmedcentral.nih.gov/articlerender.fcgi?

artid=3087812\&tool=pmcentrez\&rendertype=abstract 